\title{
Numerical Simulation Analysis on the Casing Strength under High pressure gas injection and production
}

\author{
Baohui Wang ${ }^{1, a}$, Xiangzhen Yan ${ }^{2, b}$, XiujuanYang ${ }^{3, c}$ \\ ${ }^{1}$ Architectural Engineering Institute, Weifang University, Weifang 261061, China \\ ${ }^{2}$ College of Pipeline and Civil Engineering, China University of Petroleum, Qingdao 266555, China \\ ${ }^{3}$ College of Pipeline and Civil Engineering, China University of Petroleum, Qingdao 266555, China \\ a email: 7353wbh@163.com, bemail: yanxzh@163.com, email: yangxj999@163.com
}

Keywords: high pressure; casing; strength; numerical simulation

\begin{abstract}
The statics mechanic model of the casing-cement-formation is established in this paper. Effects of formation and cement characteristics on casing strength are studied under injection-production condition. The examples show that the maximum stress occurs in the internal wall of the casing, along the orientation of minimum horizontal stress. The casing maximum strength increase with non-uniform in-situ stress coefficient and formation poisson ratio, whereas it decrease with formation elastic modulus. However, The casing maximum strength is equidirectional with the increase of cement elastic modulus and poisson ratio, whereas it decreases with cement elastic modulus ,poisson ratio and cement thickness, they have only minor impacts on casing maximum strength when they are more than 30GPa ,0.3 and 30mm respectively.
\end{abstract}

\section{Introduction}

With the rapid growth of the natural gas industry, UGS has grown to become a large and essential part of the natural gas delivery system. Long-term demand variations of natural gas caused by the increased fuel need for space heating during the cold weather require large amounts to be stored[1 4]. Most of the underground gas storage reservoirs are built up in depleted gas reservoir in the world. With the advantages of less investment, quick working effects and reclaiming investment quickly, the underground gas storage reservoirs built in depleted gas reservoir are applied widely[5 6]. However, the injection-production well is different from the conventional gas well because of its high pressure. In this paper, the statics mechanic model of the casing-cement-formation is established at the condition of gas injection-production with high pressure. The casing strength is analyzed by the finite element software. And the effects of formation and cement characteristics on casing strength are studied under high pressure gas injection and production.

\section{Establishment of the Finite Element Model of Casing-Cement-Formation}

The model is established based on the API P110 casing pipe often used in depleted gas reservoir.

The minimum yield strength of the API P110 casing pipe is 758MPa. The maximum yield strength of casing pipe is $965 \mathrm{MPa}$. Its tensile strength is $862 \mathrm{MPa}$ with wall thickness of $10.36 \mathrm{~mm}$. The injection-production condition and the formation and cement characteristics are shown in table 1 and table 2.

Table 1. The injection-production working conditions.

\begin{tabular}{|c|c|c|c|c|c|c|}
\hline Working conditions & $\begin{array}{l}\text { Diameter } \\
\text { /mm }\end{array}$ & $\begin{array}{l}\text { External diameter } \\
\text { /mm }\end{array}$ & $\begin{array}{l}\text { Wall thickness } \\
\text { /mm }\end{array}$ & $\begin{array}{c}\text { Bottom hole } \\
\text { pressure } \\
\text { /MPa }\end{array}$ & \multicolumn{2}{|c|}{$\begin{array}{l}\text { In-situ Stress } \\
\text { /MPa }\end{array}$} \\
\hline gas injection & 157.1 & 177.8 & 10.36 & 29 & maximum & 64.5 \\
\hline gas production & 157.1 & 177.8 & 10.36 & 15 & minimum & 43.5 \\
\hline
\end{tabular}


Table 2. The formation and cement characteristics.

\begin{tabular}{cccc}
\hline Name & E/MPa & Density $\mathrm{kg} / \mathrm{m}^{3}$ & $\mu$ \\
\hline casing pipe & 210000 & 7850 & 03 \\
cement & 25000 & 1830 & 0.25 \\
formation & 20000 & 2300 & 0.18 \\
\hline
\end{tabular}

The length model of casing-cement-formation is $10 \mathrm{~m}$ and the solid 95 element is used in this paper. The numerical model is established as follow:
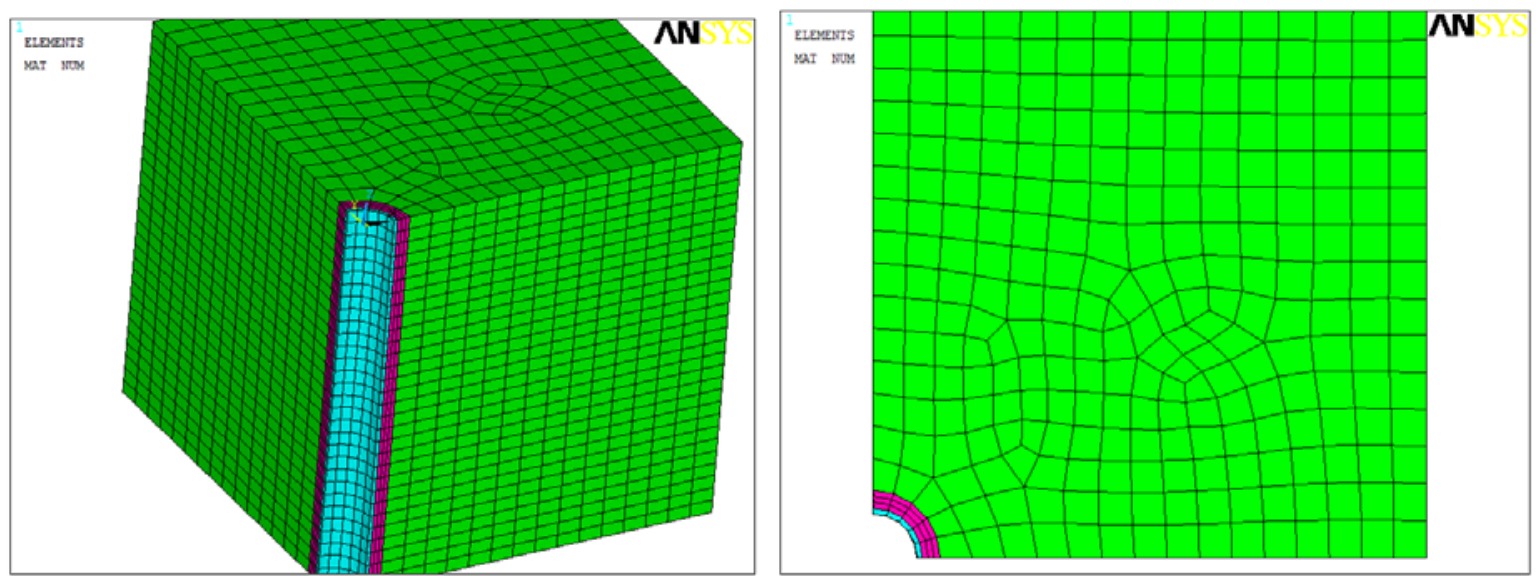

Figure 1. Numerical model.

\section{Analysis of Results}
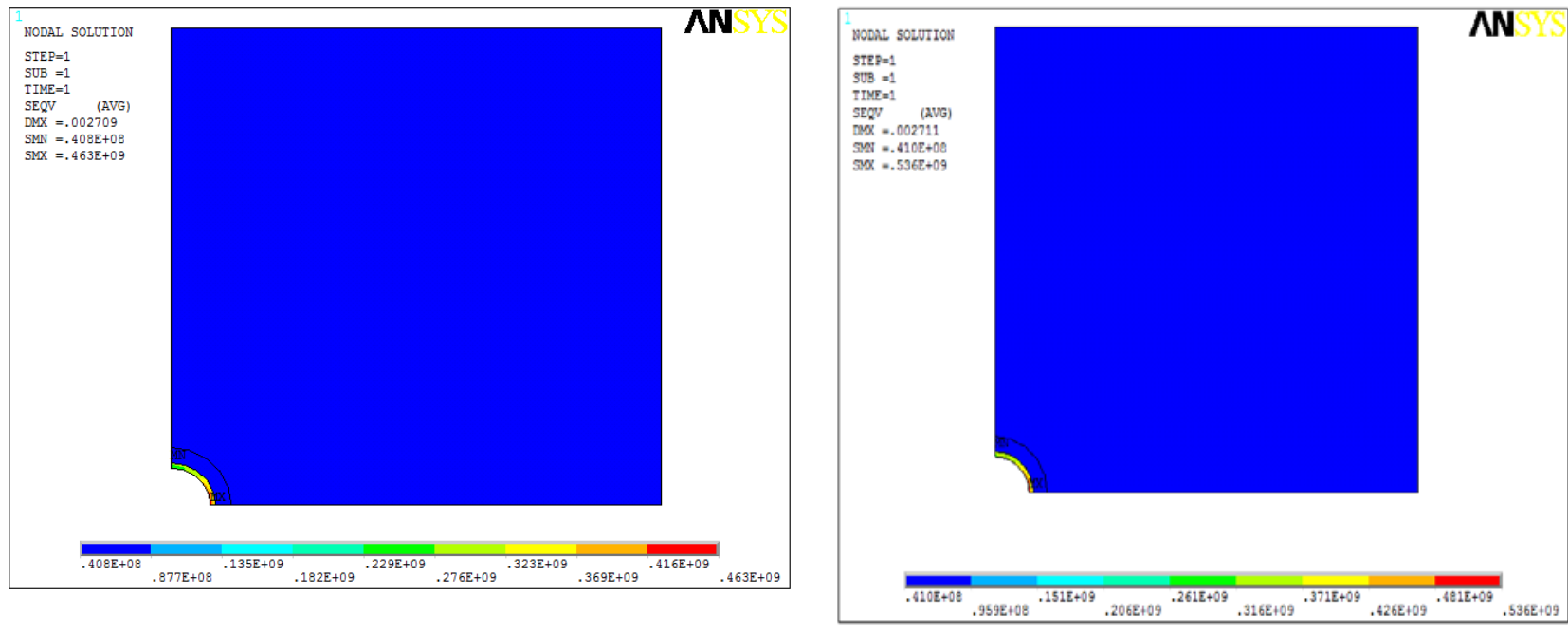

Figure 2. The effective stress distribution map

According to figure2, the position of the maximum effective strength occurred in casing inwall with the injection-production working conditions, along the orientation of minimum horizontal stress. The maximum effective strength is respectively $463 \mathrm{MPa}$ and $536 \mathrm{MPa}$ in the process of gas production and injection.

\section{The Effects of Formation and Cement Characteristics on Casing Strength}

Bsed on the Numerical model, the effect of the formation elastic modulus, formation poisson ratio, non-uniform in-situ stress coefficient, cement elastic modulus, poisson ratio and cement thickness are calculated, and then the various parameters which effect the casing strength are analyzed. The calculation results are shown in figure $3 \sim$ figure 4. 

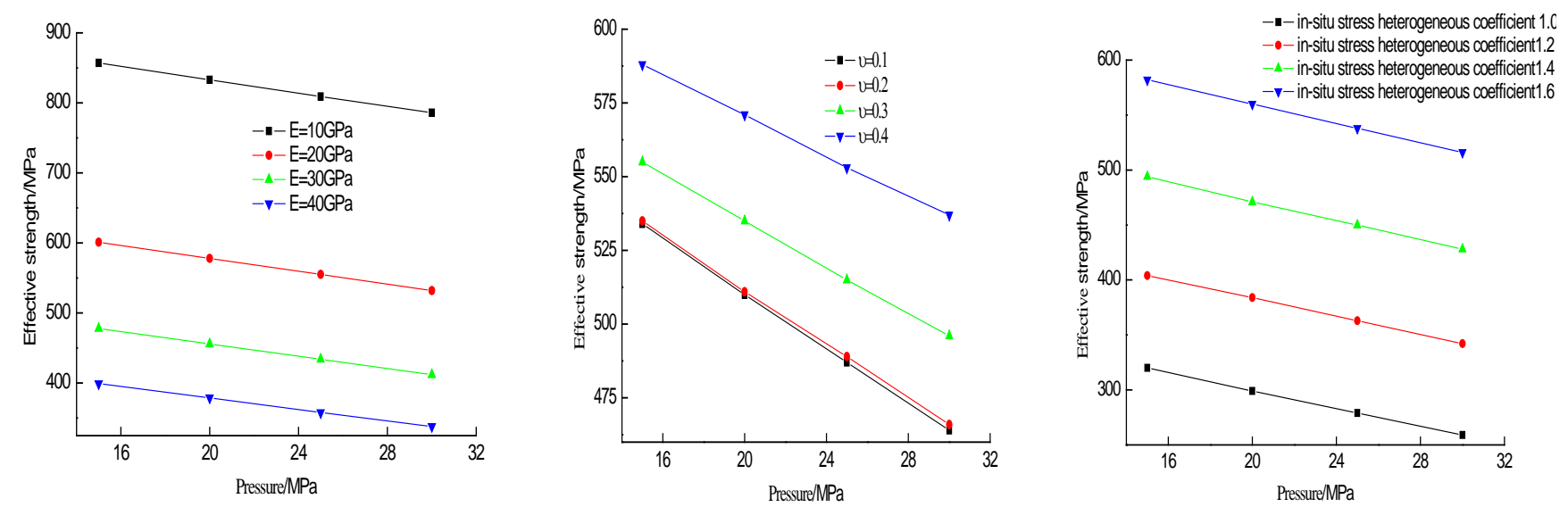

Figure 3. The Effects of Formation Characteristics on Casing Strength
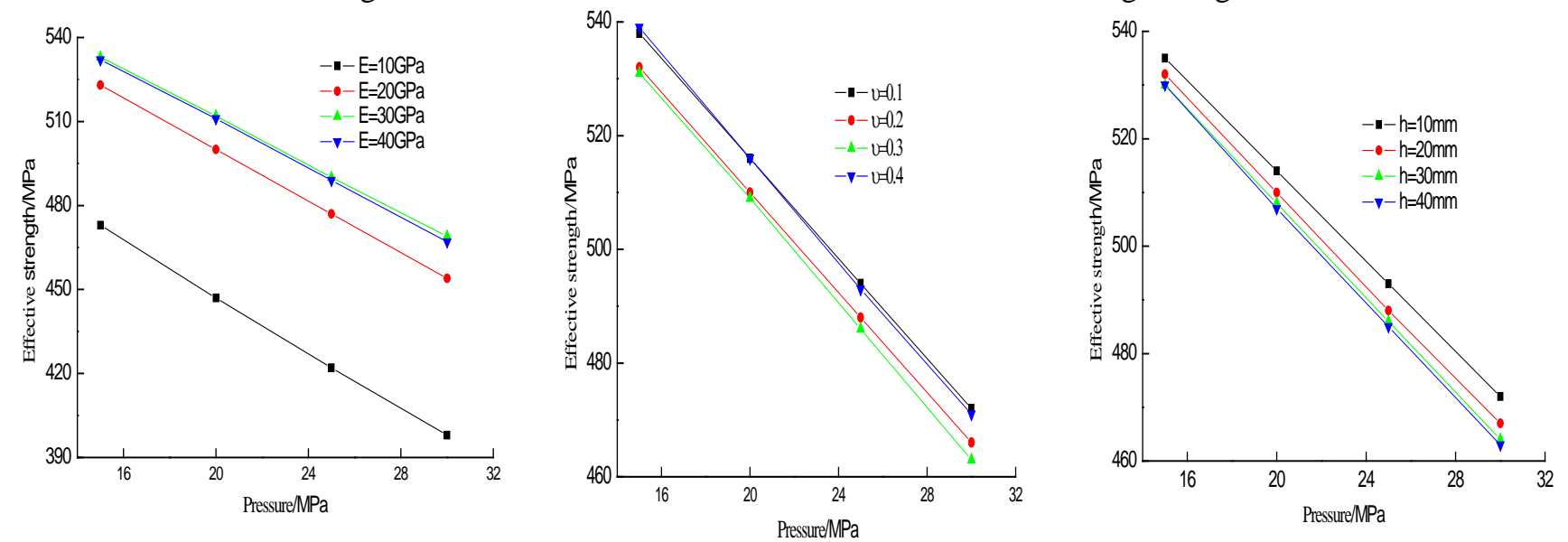

Figure 4. The Effects of Cement Characteristics on Casing Strength

As can be seen from figure 3 , the casing maximum strength decreases with formation elastic modulus in the range of 10GPa 40GPa, $400 \mathrm{MPa}$ decreasing amplitude approximately while other parameters were not changed. The casing maximum strength increases with formation poisson ratio in range of 0.1 to $0.4,54 \mathrm{MPa}$ increasing amplitude approximately while other parameters were not changed. The casing maximum strength increases with non-uniform in-situ stress coefficient in the range of 1.0 1.6, $262 \mathrm{MPa}$ increasing amplitude approximately while other parameters were not changed.

As can be seen from figure 4, the casing maximum strength increases with cement elastic modulus in the range of 10GPa 30GPa, 160MPa increasing amplitude approximately while other parameters were not changed, but it is almost unchanged as cement elastic modulus rises in the range of 30GPa 40GPa.

The casing maximum strength decreases with cement poisson ratio in range of 0.1 to $0.3,7 \mathrm{MPa}$ decreasing amplitude approximately while other parameters were not changed, but it increases as poisson ratio rises in range of 0.3 to $0.4,8 \mathrm{MPa}$ increasing amplitude approximately.

The casing maximum strength decreases with cement thickness in the range of $10 \mathrm{~mm} 30 \mathrm{~mm}$, $5 \mathrm{MPa}$ decreasing amplitude approximately while other parameters were not changed, but it is almost unchanged as cement elastic modulus rises in the range of $30 \mathrm{~mm} \sim 40 \mathrm{~mm}$.

\section{Conclusion}

The statics mechanic model of the casing-cement-formation is established in this paper. Effects of formation and cement characteristics on casing strength are studied under injection-production condition. The examples show that the maximum stress occurs in the internal wall of the casing, along the orientation of minimum horizontal stress. The casing maximum strength increase with non-uniform in-situ stress coefficient and formation elastic modulus, whereas it decrease with formation elastic modulus. However, The casing maximum strength is equidirectional with the 
increase of cement elastic modulus and poisson ratio, whereas it decreases with cement elastic modulus ,poisson ratio and cement thickness, they have only minor impacts on casing maximum strength when they are more than 30GPa ,0.3 and 30mm respectively.

\section{Acknowledgment}

The authors are very much indebted to the the National Natural Science Foundation of China (Grant No. 51274231).

\section{References}

[1] Aminan, K., Bannon, A., and Ameri, S. Gas storage in a depleted gas /condensate reservoir in the Appalachian basin. SPE: 104555, 2006.

[2] Bennion,D.B.,Thomas,F.B.,Ma,t.,and Imer. Detailed protocol for the screening and selection gas storage reservoir. SPE: 59738, 2000.

[3] Kanaga Dharmananda, Neil Kingsbury, Corrs Chambers Westgarth. Underground gas storage: issues beneath the surface. SPE: 88491-MS, 2004.

[4] David Evans, Mike Stephenson, Richard Shaw. The present and future use of land below ground[J]. Land Use Policy, 2009, 26(1):84-94

[5] S.Cantini, W.Klopf, R.Revelant, et al. Integrated log interpretation approach for underground gas storage characterization. SPE:131536-MS, 2010

[6] Stefopoulos,E.K, Damigos,D.G. Design of emergency ventilation system for an underground storage facility[J].Tunnelling and Underground Space Technology, 2007, 22(3):293-302. 